\title{
3D GEOMETRIC MOMENT INVARIANTS FROM THE POINT OF VIEW OF THE CLASSICAL INVARIANT THEORY
}

\author{
L. BEDRATYUK
}

\begin{abstract}
The aim of this paper is to clear up the problem of the connection between the $3 \mathrm{D}$ geometric moments invariants and the invariant theory, considering a problem of describing of the 3D geometric moments invariants as a problem of the classical invariant theory. Using the remarkable fact that the groups $S O(3)$ and $S L(2)$ are locally isomorphic, we reduced the problem of deriving 3D geometric moments invariants to the well-known problem of the classical invariant theory. We give a precise statement of the 3D geometric invariant moments computation, introducing the notions of the algebras of simultaneous 3D geometric moment invariants, and prove that they are isomorphic to the algebras of joint $S L(2)$-invariants of several binary forms. To simplify the calculating of the invariants we proceed from an action of Lie group $S O(3)$ to equivalent action of the Lie algebra $\mathfrak{s l}_{2}$. The author hopes that the results will be useful to the researchers in the fields of image analysis and pattern recognition.
\end{abstract}

\section{INTRODUCTION}

The issue of the $3 \mathrm{D}$ geometric moments is a generalization of the $2 \mathrm{D}$ geometric moment invariants which are widely used as global feature descriptors in the different applications for pattern recognition and image analysis. Notice, that by invariance we mean the invariance with respect to translations, uniform scaling and rotations. In nowadays, the interest to the usage of the 3D moment invariants is stimulated by the rapid growth of the 3D technologies, [1]-[4].

For the first time, the 3D moment invariants of the second order were derived in the paper [5]. In [6], Lo and Don found twelve invariants of the third order, but as it was shown in [7] there are several interdepended among them. In the book [8], the author derived 13 invariants and stated that they generate all 3D geometric moments of the third order. Finally, in [9] a set of one 1185 invariants up to order 16 was presented, but these invariants do not form a minimal generating system. However, finding a minimal generating system of the $3 \mathrm{D}$ geometric moment invariants still remains an open problem. This kind of problems turn out to be a purely algebraic questions which were studied widely in the 19th century.

Today, there exists a huge massive of the literature on the $3 \mathrm{D}$ geometric moments invariants, but a big amount of it is devoted to the application of the invariants, along with the different ways of their constructions which sometimes are rather elegant and ingenious. For instance, the methods of the quantum mechanics used in [6], [7] and [10] are very impressive.

But, those methods based on the rotation group $S O(3)$ are quite complicated and are not adapted well for the invariants calculations. In this paper, we propose to proceed from the usage of the $S O(3)$ group to the usage of its locally isomorphic group $S L(2)$. As far as the Lie algebras $\mathfrak{s o}_{3}$ and $\mathfrak{s l}_{2}$ are isomorphic, the problem of finding of $S O(3)$-invariants is equivalent to the problem of finding of $S L(2)$-invariants. The latter one is a well-known problem of the classical invariant theory issues, concequentely, the standard classical invariant theory approaches can be applied.

The aim of this paper is to consider the problem of describing 3D geometric moment invariants precisely as a problem of the classical invariant theory. We formulated the problem of the computation of the 3D geometric moments invariants based on the notion of the algebras of 
the both rational and polynomial simultaneous invariants of several binary forms. Our goal is not to find new invariants, we just put together some facts about the geometric 3D moments and presented it from a single point of view.

In this article, we proved that the introduced algebras of the 3D geometric moment invariants are isomorphic to the well-known objects of the classical invariant theory, namely, algebras of the joint invariants of the several binary forms. In the rational case, we firstly applied the standard infinitesimal method to the studying of the geometric moments and reduced the problem of calculating the $S O(3)$-invariants to the equivalent problem of calculating the invariants of its Lie algebra $\mathfrak{s o}_{3}$.

The paper is arranged as follows.

In Sect. 2, we review basic concepts of the classical invariant theory and provide the necessary facts regarding the action of the Lie groups $S O(3)$ and $S L(2)$ and their Lie algebras $\mathfrak{s o}_{3}$, and $\mathfrak{s l}_{2}$, respectively on the vector spaces of binary and ternary forms. We introduce the notions of the algebras of simultaneous rational and polynomial 3D geometric moment invariants and prove that they are isomorphic to the algebras of joint rational and polynomial $\mathfrak{s l}_{2}$-invariants of several binary forms. Also, we presented a system of partial differential equations concerning those invariants.

In Sect. 3, we recall the basic notions of the representation theory of the Lie algebras and present a minimal generating system for the algebra of the $3 \mathrm{D}$ geometric polynomial moments invariants of orders two and three which is expressed in the terms of eigenvectors of the Casimir operator. Also we derive the formula for the corresponding Poincaré series.

In Sect. 4, we count out the number of elements in a minimal generating set of the algebra rational rotation invariants and present such minimal generating set for the rational invariants of second and third orders. Also, we express the explicit form the invariants of the degrees one of arbitrary order.

The article is a continuation of the [12] article, which addresses the similar issues for the 2D geometric moment invariants.

\section{Preliminary Concepts}

In this section, we briefly review some basic concepts of the classical invariant theory, give the necessary facts about the Lie groups $S O(3), S L(2)$ and their Lie algebras $\mathfrak{s o}_{3}$ and $\mathfrak{s l}_{2}$. Also, we give the definition of the algebras of simultaneous rational and polynomial 3D geometric moment invariants and then establish an isomorphism between these algebras and the algebras of the joint invariants of several binary forms.

2.1. Basic notions of the invariant theory. Let $G L(V)$ be the group of all invertible linear transformations of a finite-dimensional complex vector space $V$. The natural action of $G L(V)$ on $V$ produces an action on the algebras of polynomial and rational functions $\mathbb{C}[V]$ and $\mathbb{C}(V)$. If $g \in G L(V), F \in \mathbb{C}[V]$ define a new polynomial function $g \cdot F \in \mathbb{C}[V]$ by

$$
(g \cdot F)(v)=F\left(g^{-1} v\right) .
$$

If $G$ is subgroup of $G L(V)$ we say that $F$ is $G$-invariant if $g \cdot F=F$ for all $g \in G$. The $G$ invariant polynomial functions forms a subalgebra $\mathbb{C}[V]^{G}$ of $\mathbb{C}[V]$. The algebra $\mathbb{C}[V]^{G}$ is called the algebra of the polynomial $G$-invariants. In the similar way, we define the algebra of rational invariants $\mathbb{C}(V)^{G}$.

Let us recall that a derivation of an algebra $R$ is an additive map $L$ satisfying the Leibniz rule:

$$
L\left(r_{1} r_{2}\right)=L\left(r_{1}\right) r_{2}+r_{1} L\left(r_{2}\right), \text { for all } r_{1}, r_{2} \in R
$$


The subalgebra

$$
\operatorname{ker} L:=\{f \in R \mid L(f)=0\},
$$

is called the kernel of the derivation $L$.

Let now $G$ be a simply connected Lie group acting on $V$ and let $\mathfrak{g}$ be its Lie algebra. By an action of $\mathfrak{g}$ we understand its representation by preserving Lie products of linear operators on $V$. We will extend these operators on $\mathbb{C}[V]$ and $\mathbb{C}(V)$ as derivatives. It is well known that the condition $I \in \mathbb{C}[V]^{G}$ is equivalent to $L(I)=0, \forall L \in \mathfrak{g}$. Thus,

$$
\mathbb{C}[V]^{G}=\mathbb{C}[V]^{\mathfrak{g}}=\bigcap_{L \in \mathfrak{g}} \operatorname{ker} L .
$$

As a linear object, a Lie algebra is often a much easier to work with than working directly with the corresponding Lie group. We will use this fact later to ease the computation of invariants.

The classical invariant theory is focused on the action of the general linear group on homogeneous polynomials, with an emphasis on the forms, mainly binary and ternary ones. Let us consider two important invariant constructions which illustrate a computational advantage of the Lie algebras techniques.

Example 2.1. The space $V_{d}$ of binary forms of degree $d$ is the vector space:

$$
V_{d}=\left\{\sum_{k=0}^{d}\left(\begin{array}{l}
d \\
k
\end{array}\right) a_{k} x^{d-k} y^{k} \mid a_{k} \in \mathbb{C}\right\} .
$$

The goup $S L(2)$ is a group of $2 \times 2$ complex matrices with determinant one. The corresponding Lie algebra $\mathfrak{s l}_{2}$ is generated by the matrices with zero trace

$$
h=\left(\begin{array}{cc}
1 & 0 \\
0 & -1
\end{array}\right), \quad e_{+}=\left(\begin{array}{ll}
0 & 1 \\
0 & 0
\end{array}\right), \quad e_{-}=\left(\begin{array}{ll}
0 & 0 \\
1 & 0
\end{array}\right)
$$

and the following commutation relations

$$
\left[h, e_{+}\right]=2 e_{+}, \quad\left[h, e_{-}\right]=-2 e_{-}, \quad\left[e_{+}, e_{-}\right]=h .
$$

The elements $e_{-}, e_{+}, h$ act on $V_{d}$ by the derivations

$$
-y \frac{\partial}{\partial x},-x \frac{\partial}{\partial y},-x \frac{\partial}{\partial x}-y \frac{\partial}{\partial y}
$$

and act on $\mathbb{C}\left(V_{d}\right)$ by the derivations

$$
D_{+}=\sum_{k=0}(d-1) a_{k+1} \frac{\partial}{a_{k}}, D_{-}=\sum_{k=1}^{d} k a_{k-1} \frac{\partial}{a_{k}}, H=\sum_{k=0}^{d}(d-2 k) a_{k} \frac{\partial}{a_{k}} .
$$

The polynomial solutions of the corresponding system of differential equations generate the algebra $\mathbb{C}\left[V_{d}\right]^{\mathfrak{s l}_{2}}$ of invariants of binary form. Since,

$$
\left[D_{+}, D_{-}\right]=D_{+} D_{-}-D_{-} D_{+}=H
$$

it follows that

$$
\mathbb{C}\left[V_{d}\right]^{\mathfrak{s l}_{2}}=\operatorname{ker} D_{+} \cap \operatorname{ker} D_{-} .
$$

The minimal generating systems of $\mathbb{C}\left[V_{d}\right]^{\mathfrak{s l}_{2}}$ were a major object of research in classical invariant theory of the 19th century. At present, such generators have been found only for $d \leq 10$.

In the similar manner we define an action of $S L(2)$ and $\mathfrak{s l}_{2}$ on the direct sum

$$
W=V_{k_{1}} \oplus V_{k_{2}} \oplus \cdots \oplus V_{k_{n}}
$$


The corresponding algebras of polynomial and rational invariants are called the algebras of joint invariants (polynomial or rational) of binary forms and denoted by $\mathbb{C}[W]^{\mathfrak{s o}_{2}}$ and $\mathbb{C}(W)^{\mathfrak{s o}_{2}}$, respectively. At the present time, the algebras of the joint invariants are only known for a few values of $k_{1}, k_{2}, \ldots, k_{n}$, see [13].

Example 2.2. The $3 \mathrm{D}$ rotation group $S O(3)$ is the group of all rotations about the origin of three-dimensional Euclidean space. It is a three-parameters group with the following matrix realization

$$
\left(\begin{array}{ccc}
\cos \varphi & -\sin \varphi & 0 \\
\sin \varphi & \cos \varphi & 0 \\
0 & 0 & 1
\end{array}\right),\left(\begin{array}{ccc}
\cos \theta & 0 & -\sin \theta \\
0 & 1 & 0 \\
\sin \theta & 0 & \cos \theta
\end{array}\right),\left(\begin{array}{ccc}
\cos \psi & -\sin \psi & 0 \\
\sin \psi & \cos \psi & 0 \\
0 & 0 & 1
\end{array}\right), \psi, \theta, \varphi \in[0,2 \pi]
$$

where the parameters $\psi, \theta, \varphi$ are the Euler angles.

The associated tree-dimensional complex Lie algebra $\mathfrak{s o}_{3}$ is generated by the matrix

$$
e_{1}=\left(\begin{array}{ccc}
0 & 1 & 0 \\
-1 & 0 & 0 \\
0 & 0 & 0
\end{array}\right), e_{2}=\left(\begin{array}{ccc}
0 & 0 & 1 \\
0 & 0 & 0 \\
-1 & 0 & 0
\end{array}\right), e_{3}=\left(\begin{array}{ccc}
0 & 0 & 0 \\
0 & 0 & 1 \\
0 & -1 & 0
\end{array}\right)
$$

and the Lie brackets are given by commutator, i.e.,

$$
\left[e_{1}, e_{2}\right]=-e_{3},\left[e_{1}, e_{3}\right]=e_{2},\left[e_{2}, e_{3}\right]=-e_{1} .
$$

Let us recall that the space of ternary forms of degree $d$ is the vector space:

$$
T_{d}=\left\{\sum_{j+k+l=d}\left(\begin{array}{c}
d \\
j, k, l
\end{array}\right) a_{j, k, l} x^{j} y^{k} z^{l} \mid a_{j, k, l} \in \mathbb{C}\right\},
$$

where $\left(\begin{array}{c}d \\ j, k, l\end{array}\right)=\frac{d !}{j ! k ! l !}$ denotes the multinomial coefficient. The linear functions

$$
\sum_{j+k+l=d}\left(\begin{array}{c}
d \\
j, k, l
\end{array}\right) a_{j, k, l} x^{j} y^{k} z^{l} \mapsto a_{j, k, l}
$$

form a basis of the dual vector space $T_{d}^{*}$. For convenience, it is useful to equal the functions and the corresponding coefficients $a_{j, k, l}$.

It is a well-known, see, for example, [14], that $\mathfrak{s o}_{3}$ acts on $T_{d}$ by derivations

$$
x \frac{\partial}{\partial y}-y \frac{\partial}{\partial x}, x \frac{\partial}{\partial z}-z \frac{\partial}{\partial x}, y \frac{\partial}{\partial z}-z \frac{\partial}{\partial y} .
$$

Wherefrom, it follows that $\mathfrak{s o}_{3}$ acts on the dual space $T_{d}^{*}$ by the derivations:

\section{Theorem 1.}

$$
\begin{aligned}
& E_{1}\left(a_{j, k, l}\right)=k a_{j+1, k-1, l}-j a_{j-1, k+1, l}, \\
& E_{2}\left(a_{j, k, l}\right)=l a_{j+1, k, l-1}-j a_{j-1, k, l+1}, \\
& E_{3}\left(a_{j, k, l}\right)=l a_{j, k+1, l-1}-k a_{j, k-1, l+1} .
\end{aligned}
$$

Proof. Using a definition of the $\mathfrak{s o}_{3}$-action on the dual space.

In the similar manner, we define an action of $S O(3)$ and $\mathfrak{s o}_{3}$ on the direct sum

$$
U=T_{k_{1}} \oplus T_{k_{2}} \oplus \cdots \oplus T_{k_{3}} .
$$


The corresponding algebras of polynomial and rational invariants are called the algebras of joint $3 D$ rotation invariants and denoted by $\mathbb{C}[U]^{\mathfrak{s o}_{3}}$ and $\mathbb{C}(U)^{\mathfrak{s o}_{3}}$, respectively. More details about 3D rotations can be found, e.g., in [14], [15].

An important detail that plays a crucial role in this article is a well-known fact that the complex Lie algebras $\mathfrak{s o}_{3}$ and $\mathfrak{s l}_{3}$ are isomorphic, although the corresponding Lie groups are not isomorphic. To establish the isomorphism, we introduce new matrices

$\mathcal{D}_{+}=i e_{1}+e_{2}=\left(\begin{array}{ccc}0 & i & 1 \\ -i & 0 & 0 \\ -1 & 0 & 0\end{array}\right), \mathcal{D}_{-}=i e_{1}-e_{2}=\left(\begin{array}{ccc}0 & i & 1 \\ -i & 0 & 0 \\ -1 & 0 & 0\end{array}\right), \mathcal{H}=2 i e_{3}=2 i\left(\begin{array}{ccc}0 & 0 & 0 \\ 0 & 0 & 1 \\ 0 & -1 & 0\end{array}\right)$.

By direct calculations of their commutators, we obtain

$$
\left[\mathcal{H}, \mathcal{D}_{+}\right]=2 \mathcal{D}_{+}, \quad\left[\mathcal{H}, \mathcal{D}_{-}\right]=-2 \mathcal{D}_{-}, \quad\left[\mathcal{D}_{+}, \mathcal{D}_{-}\right]=\mathcal{H}
$$

The commutators coincide with the corresponding commutators of the basic elements for the algebra $\mathfrak{s l}_{2}$, which establishes the isomorphism.

Note that the operators act on the basis elements of $T_{d}^{*}$ as follows

$$
\begin{aligned}
& \mathcal{D}_{+}\left(a_{j, k, l}\right)=i\left(k a_{j+1, k-1, l}-j a_{j-1, k+1, l}\right)+l a_{j+1, k, l-1}-j a_{j-1, k, l+1}, \\
& \mathcal{D}_{-}\left(a_{j, k, l}\right)=i\left(k a_{j+1, k-1, l}-j a_{j-1, k+1, l}\right)-\left(l a_{j+1, k, l-1}-j a_{j-1, k, l+1}\right), \\
& \mathcal{H}\left(a_{j, k, l}\right)=2 i\left(l a_{j, k+1, l-1}-k a_{j, k-1, l+1}\right) .
\end{aligned}
$$

As we will see later, this isomorphism allows us reduce the problem of finding the $3 \mathrm{D}$ rotation invariants to the problem of calculating the invariants of binary forms which is a classical invariant theory problem.

2.2. Algebras of $3 \mathrm{D}$ rotation invariants. In the sequel, we will work with the similarity transformation group $G$ which is widely used in 3D image analysis and pattern recognition. The group is the semi-direct product of the space translation group $T R(3)$, the direct product of the space rotation group $S O(3)$ and the uniform scaling group $\mathbb{R}^{*}$ :

$$
G=\left(\mathbb{R}^{*} \times S O(3)\right) \rtimes T R(3) .
$$

The introduction of the notion of $2 \mathrm{D}$ image moment invariants by $\mathrm{Hu}$ in the significant paper [11] is a vivid example of the application of the classical invariant theory to the pattern recognition. A way of the generalization of this approach for $3 \mathrm{D}$ images was suggested in [5], [6]. Let $\boldsymbol{F}$ be a set of real finite piece-wise continuous functions that can have nonzero values only in a compact subset of $\mathbb{R}^{3}$.

Let us consider the geometric moments of $f \in \boldsymbol{F}$

$$
m_{p q r}(f(x, y, z))=m_{p q r}=\iiint_{\Omega} x^{p} y^{q} z^{r} f(x, y, z) d x d y d z, \Omega \subset \mathbb{R}^{3},
$$

and the central geometric moment

$$
\mu_{p q r}(f(x, y, z))=\mu_{p q r}=\iiint_{\Omega}(x-\bar{x})^{p}(y-\bar{y})^{q}(z-\bar{z})^{r} f(x, y, z) d x d y d z
$$

where

$$
\bar{x}=\frac{m_{100}}{m_{0,0,0}}, \bar{y}=\frac{m_{010}}{m_{0,0,0}}, \bar{y}=\frac{m_{001}}{m_{0,0,0}}
$$


The central geometric moments are already invariants under the translation group. After the normalization

$$
\eta_{p, q, r}=\frac{\mu_{p, q, r}}{\mu_{0,0}^{1+\frac{p+q+r}{3}}}, p+q+r \geq 2
$$

they become invariants of the scaling group. Therefore, the problem of determining of the 3D geometric image moment invariants can be reduced to the problem of finding $S O(3)$-invariants as functions of the normalized central geometric moments. Therefore, in this paper we will deal only with the normalized $S O(3)$-invariant functions.

We will consider two types of such functions, specifically, polynomials and rational ones. Let $\mathbb{C}[\eta]$ and $\mathbb{C}(\eta)$ be the polynomial and rational algebras in countably many variables $\left\{\eta_{p, q, r}\right\}_{p+q+r=2}^{\infty}$ considered with the natural action of the group $S O(3)$. Denote by $\mathbb{C}[\eta]^{S O(3)}$ and $\mathbb{C}(\eta)^{S O(3)}$ the corresponding algebras of polynomial and rational moment invariants, respectively. Since these algebras are not finitely generated, then a complete set of invariants consists of infinitely many invariants. However, these algebras can be approximated by the finitely generated algebras $\mathbb{C}[\eta]_{d}^{S O(3)}$ and $\mathbb{C}(\eta)_{d}^{S O(3)}$ where $[\eta]_{d}=\left\{\eta_{p, q, r}, 2 \leq p+q+r \leq d\right\}$. The elements of these algebras are called the simultaneous 3D geometric moment (polynomial or rational) invariants of order up to $d$. For instance, the invariant

$$
\eta_{2,0,0}+\eta_{0,2,0}+\eta_{0,0,2}
$$

belong to $\mathbb{C}[\eta]_{2}^{S O(3)}$ and $\mathbb{C}(\eta)_{2}^{S O(3)}$.

Remarkably, in general case, the problem of describing the algebras of the simultaneous 3D geometric moment invariants can be reduced to the well-known problems of the classical invariant theory. It turns out that the algebras $\mathbb{C}(\eta)_{d}^{S O(3)}$ and $\mathbb{C}(\eta)_{d}^{S O(3)}$ are isomorphic to the algebras of joint polynomial and rational $S L(2)$-invariants of some system of binary forms.

The locally isomorphism of $S O(3)$ and $S L(2)$ implies the following theorem.

Theorem 2. The algebras of polynomial and rational simultaneous $3 D$ geometric moment invariants $\mathbb{C}[\eta]_{d}^{S O(3)}$ and $\mathbb{C}(\eta)_{d}^{S O(3)}$ are isomorphic to the algebras of invariants $\mathbb{C}\left[U_{d}\right]^{\mathfrak{s l}_{2}}$ and $\mathbb{C}\left(U_{d}\right)^{\mathfrak{s l}_{2}}$, respectively. Here

$$
U_{d}=T_{2} \oplus T_{3} \oplus \cdots \oplus T_{d}
$$

and $T_{k}$ is the vector space of ternary forms of order $k$.

Proof. It is sufficient to check that the algebras $\mathfrak{s o}_{3}$ and $\mathfrak{s l}_{2}$ act by identical derivatives. Let us consider the action of the element

$$
\left(\begin{array}{ccc}
\cos \theta & -\sin \theta & 0 \\
\sin \theta & \cos \theta & 0 \\
0 & 0 & 1
\end{array}\right) \in S O(3)
$$

on the normalized moment $\eta_{j, k, l}$. By the definition, we have 


$$
\begin{gathered}
\left(\begin{array}{ccc}
\cos \theta & -\sin \theta & 0 \\
\sin \theta & \cos \theta & 0 \\
0 & 0 & 1
\end{array}\right)^{-1} \eta_{p, q, r}=\iiint_{\Omega}(x \cos \theta-y \sin \theta)^{p}(x \sin \theta+y \cos \theta)^{q} z^{r} f(x, y, z) d x d y d z= \\
=\iiint_{\Omega} \sum_{k=0}^{p} \sum_{j=0}^{q}(-1)^{p-k}\left(\begin{array}{l}
p \\
k
\end{array}\right)\left(\begin{array}{l}
q \\
j
\end{array}\right)(\cos \theta)^{p-k+j}(\sin \theta)^{q+k-j} x^{p-k+q-j} y^{k+j} z^{r} f(x, y, z) d x d y d z= \\
=\sum_{k=0}^{p} \sum_{j=0}^{q}(-1)^{p-k}\left(\begin{array}{l}
p \\
k
\end{array}\right)\left(\begin{array}{l}
q \\
j
\end{array}\right)(\cos \theta)^{p-k+j}(\sin \theta)^{q+k-j} \eta_{p-k+q-j, k+j, r} .
\end{gathered}
$$

To get the action of the Lie algebra $\mathfrak{s o}_{3}$ we differentiate it by $\theta$ and, after simplification, we obtain:

$$
\left.\frac{d}{d \theta}\left(\begin{array}{ccc}
\cos \theta & -\sin \theta & 0 \\
\sin \theta & \cos \theta & 0 \\
0 & 0 & 1
\end{array}\right)^{-1} \eta_{p, q, r}\right|_{\theta=0}=q \eta_{p+1, q-1, r}-p \eta_{p-1, q+1, r}
$$

It is easy to see that this action is identical to the derivation $E_{1}$, as it described in Example 2.2. In the same manner, we can show that the following elements of $S O(3)$ :

$$
\left(\begin{array}{ccc}
1 & 0 & 0 \\
0 & \cos \theta & -\sin \theta \\
0 & \sin \theta & \cos \theta
\end{array}\right),\left(\begin{array}{ccc}
\cos \psi & -\sin \psi & 0 \\
\sin \psi & \cos \psi & 0 \\
0 & 0 & 1
\end{array}\right)
$$

act like the derivations $E_{2}$ and $E_{3}$. Thus, the normalized $3 \mathrm{D}$ geometric moment invariants and the joint $\mathfrak{s o}_{3}$-invariants of the binary forms are defined by the same system of the partial differential equation. It implies that $\mathbb{C}(\eta)_{d}^{S O(3)} \cong \mathbb{C}\left(U_{d}\right)^{\mathfrak{s o}_{3}}$ and $\mathbb{C}[\eta]_{d}^{S O(3)} \cong \mathbb{C}\left[U_{d}\right]^{\mathfrak{s o}_{3}}$. Since, $\mathfrak{s o}_{3} \cong \mathfrak{s l}_{2}$ we get that $\mathbb{C}(\eta)_{d}^{S O(3)} \cong \mathbb{C}\left(U_{d}\right)^{\mathfrak{s l}_{2}}$ and $\mathbb{C}[\eta]_{d}^{S O(3)} \cong \mathbb{C}\left[U_{d}\right]^{\mathfrak{s l}_{2}}$ as required.

The isomorphism has the simple form: $a_{j, k, r} \mapsto \eta_{j, k, r}$.

Thus, from the point of view of the classical invariant theory, the problem of the description of the algebras $3 \mathrm{D}$ geometric image moment invariants $\mathbb{C}[\eta]_{d}^{S O(3)}, \mathbb{C}(\eta)_{d}^{S O(3)}$ can be reduced to the following two problems.

- Problem 1. What is a minimal generating set of the algebra polynomial joint invariants $\mathbb{C}\left[U_{d}\right]^{\mathfrak{s l}_{2}}$ ?

- Problem 2. What is a minimal generating set of the algebra rational joint invariants $\mathbb{C}\left(U_{d}\right)^{\mathfrak{s l}_{2}}$ ?

Besides, the problem of deriving of 3D geometric moment invariants can be reduced to a system of differential equations. The last result of Subsest. 2.1 implies the theorem:

Theorem 3. The algebra $\mathbb{C}\left(U_{d}\right)^{\mathfrak{s l}_{2}}$ coincides with the algebra of rational solutions of the first order simultaneous partial differential equations:

$$
\left\{\begin{array}{c}
\sum_{2 \leq j+k+l \leq d}\left(k \eta_{j+1, k-1, l}-j \eta_{j-1, k+1, l}\right) \frac{\partial U}{\partial \eta_{j, k, l}}=0 \\
\sum_{2 \leq j+k+l \leq d}\left(l \eta_{j+1, k, l-1}-j \eta_{j-1, k, l+1}\right) \frac{\partial U}{\partial \eta_{j, k, l}}=0 .
\end{array}\right.
$$

In the next section we will deal with the algebras $\mathbb{C}\left[U_{d}\right]^{\mathfrak{s l}_{2}}$ and $\mathbb{C}\left(U_{d}\right)^{\mathfrak{s l}_{2}}$. 


\section{The algebra of polynomial invariants $\mathbb{C}\left[U_{d}\right]^{\mathfrak{s l}_{2}}$.}

Let us recall some facts about representations of the Lie algebra $\mathfrak{s l}_{2}$.

3.1. Representations of $\mathfrak{s l}_{2}$. Let $V$ be a finite-dimensional complex vector space equipped with non-trivial linear operators $D_{+}, D_{-}, H: V \rightarrow V$, which satisfy the following commutation relations

$$
\left[H, D_{+}\right]=H D_{+}-D_{+} H=2 D_{+}, \quad\left[H, D_{-}\right]=-2 D_{-}, \quad\left[D_{+}, D_{-}\right]=H
$$

Then $V$ is called the linear representation of the Lie algera $\mathfrak{s l}_{2}$ or $\mathfrak{s l}_{2}$-module. The vector spaces $T_{k}^{*}, U_{d}$ defined above are the samples of $\mathfrak{s l}_{2}$-modules. The modules 0 and $V$ are called trivial modules. A $\mathfrak{s l}_{2}$-module $V$ is called irreducible if $V$ has no non-trivial $\mathfrak{s l}_{2}$-submodule. All irreducible $\mathfrak{s l}_{2}$-modules, up to isomorphism, can be described with the following construction.

Let $\mathcal{V}_{n}=\left\langle a_{0}, a_{1}, \ldots a_{n}\right\rangle$ be a $n+1$-dimension complex vector space and let the linear operators $D_{-}, D_{+}, H: V_{n} \rightarrow V_{n}$ act on elements of the basis as follows :

$$
D_{-}\left(a_{k}\right)=k a_{k-1}, D_{+}\left(a_{k}\right)=(n-k) a_{k+1}, H\left(a_{k}\right)=(n-2 k) a_{k} .
$$

Let us check that the commutation relation for $\mathfrak{s l}_{2}$ are fulfilled. In fact, we have

$$
\begin{aligned}
& {\left[D_{-}, D_{+}\right]\left(a_{k}\right)=D_{-}\left(D_{+}\left(a_{k}\right)\right)-D_{+}\left(D_{-}\left(a_{k}\right)\right)=D_{-}\left((d-k) a_{k+1}\right)-D_{+}\left(k a_{k-1}\right)=} \\
& =(d-k)(k+1) a_{k}-k(d-(k-1)) a_{k}=(d-2 k) a_{k}=H\left(a_{k}\right), \\
& {\left[H, D_{-}\right]\left(a_{k}\right)=H\left(D_{-}\left(a_{k}\right)\right)-D\left(H\left(a_{k}\right)\right)=H\left(k a_{k-1}\right)-D\left((d-2 k) a_{k}\right)=} \\
& =k(d-2(k-1)) a_{k-1}-(d-2 k) k a_{k-1}=2 k a_{k-1}=2 D\left(a_{k}\right), \\
& {\left[H, D_{+}\right]\left(a_{k}\right)=H\left(D_{+}\left(a_{k}\right)\right)-D_{+}\left(H\left(a_{k}\right)\right)=H\left((d-k) a_{k+1}\right)-D_{+}\left((d-2 k) a_{k}\right)=} \\
& =(d-k)(d-2(k+1)) a_{k+1}-(d-2 k)(d-k) a_{k+1}=-2(d-k) a_{k+1}=-2 D_{+}\left(a_{k}\right) .
\end{aligned}
$$

Therefore, $\mathcal{V}_{n}$ is an representation of $\mathfrak{s l}_{2}$. The vector space $\mathcal{V}_{n}$ considered together with the indicated action of the operators $D_{-}, D_{+}, H$ is called the standard irreducible $s l_{2}$-module. It is well known, see [18], the an arbitrary $\mathfrak{s l}_{2}$-module can be decomposed into an direct sum of the irreducible standard $\mathfrak{s l}_{2}$-modules. Next, we present an algorithm of decomposing an arbitrary $\mathfrak{s l}_{2}$-module into the irreducible submodules. We use the algorithm later to construct invariants.

Let $W$ be an arbitrary $\mathfrak{s l}_{2}$-module. For any element $w \in W$ the smallest natural number, denoted $\operatorname{ord}(w)$, such that

$$
D_{+}^{\operatorname{ord}(z)}(w) \neq 0 \text {, but } D_{+}^{\text {ord }(z)+1}(w)=0 .
$$

is called the order of $w$. Since $D_{+}$is a nilpotent operator, the order $\operatorname{ord}(w)$ is defined correctly.

A vector $z \in W$ is called the lowest weight vector if the following conditions holds: $D_{-}(z)=0$ and $H(z)=\operatorname{ord}(z) z$. Any lowest weight vector defines an irreducible $s l_{2}$-module which is isomorphic to the standard $s l_{2}$-module. The following theorem holds.

Theorem 4. Suppose $z \in W$ is a lowest weight vector. Then the vector space

$$
\mathcal{V}_{s}(z):=\left\langle v_{0}(z), v_{1}(z), \ldots v_{s}(z)\right\rangle, s=\operatorname{ord}(z)
$$

where

$$
v_{k}(z)=\frac{(s-k) !}{s !} D_{+}^{k}(z), v_{0}(z):=z,
$$

is $\mathfrak{s l}_{2}$-module isomorphic to the standard sl $l_{2}$-module $\mathcal{V}_{s}$ 
Proof. It is easy to verify by direct calculations that the relations

$$
\begin{aligned}
& H\left(D_{+}^{k}(z)\right)=(s-2 k) D_{+}^{k}(z), \\
& D_{-}\left(D_{+}^{k}(z)\right)=k(s-k+1) D_{+}^{k-1}(z),
\end{aligned}
$$

hold for all $k \leq s$. Let us construct the standard $\mathfrak{s l}_{2}$-module $\mathcal{V}_{s}$ with the basis vectors of the form

$$
v_{k}=\alpha_{k} D_{+}^{k}(z), k=0, \ldots, s,
$$

for some unknown constants $\alpha_{k} \in \mathbb{C}$.

In order the vectors form a basis of $\mathcal{V}_{s}$, the following two conditions must be satisfied:

$$
D_{-}\left(v_{k}\right)=k v_{k-1}, D_{+}\left(v_{k}\right)=(s-k) v_{k+1},
$$

for all $k=0, \ldots, s$. Since

$$
D_{-}\left(v_{k}\right)=D_{-}\left(\alpha_{k} D_{+}^{k}(z)\right)=\alpha_{k} D_{-}\left(D_{+}^{k}(z)\right)=\alpha_{k} k(s-k+1) D_{+}^{k-1}(z),
$$

and

$$
D_{-}\left(v_{k}\right)=k v_{k-1}=k \alpha_{k-1} D_{+}^{k-1}(z),
$$

we obtain the recurrence equation for $\alpha_{k}$ :

$$
\alpha_{k}(s-k+1)=\alpha_{k-1}, \alpha_{0}=1 .
$$

It follows immediately that

$$
\alpha_{k}=\frac{1}{s(s-1) \ldots(s-k+1)} \alpha_{0}=\frac{(s-k) !}{s !} .
$$

Let us make sure that the second relation

$$
D_{+}\left(v_{k}\right)=(s-k) v_{k+1},
$$

also holds. We have

$$
D_{+}\left(v_{k}\right)=D_{+}\left(\alpha_{k} D_{+}^{k}\left(v_{0}\right)\right)=\frac{(s-k) !}{s !} \frac{s !}{(s-(k+1)) !} \frac{(s-(k+1)) !}{s !} D_{+}^{k+1}\left(v_{0}\right)=(s-k) v_{k+1},
$$

as required which ends the proof.

The theorem below determines a structure of the $s l_{2}$-modules $T_{d}^{*}$ and $U_{d}$ up to isomorphism:

Theorem 5. The following decompositions hold:

$$
\begin{aligned}
& T_{d}^{*} \cong \mathcal{V}_{2 d} \oplus \mathcal{V}_{2 d-4} \oplus \mathcal{V}_{2 d-8} \oplus \cdots \oplus \mathcal{V}_{2 d-4\left[\frac{d}{2}\right]} \\
& U_{d}^{*} \cong l_{0}^{(d)} \mathcal{V}_{0} \oplus l_{1}^{(d)} \mathcal{V}_{2} \oplus l_{2}^{(d)} \mathcal{V}_{4} \oplus \cdots \oplus l_{d}^{(d)} \mathcal{V}_{2 d}
\end{aligned}
$$

where

$$
l_{k}^{(d)}=\left\{\begin{array}{l}
0, \text { if } k>d, \\
{\left[\frac{d-k}{2}\right], \text { if } k=0,1} \\
{\left[\frac{d-k}{2}\right]+1, \text { if } k \geq 2 .}
\end{array}\right.
$$

Since the proof requires some advanced results of the Lie algebras representation theory, we omit the proof. 
Example 3.1. For small d we have

$$
\begin{aligned}
& U_{2}^{*}=T_{2}^{*} \cong \mathcal{V}_{0} \oplus \mathcal{V}_{4} \\
& U_{3}^{*}=T_{2}^{*}+T_{3}^{*} \cong \mathcal{V}_{0} \oplus \mathcal{V}_{2} \oplus \mathcal{V}_{4} \oplus \mathcal{V}_{6} \\
& U_{4}^{*}=T_{2}^{*}+T_{3}^{*}+T_{4}^{*} \cong 2 \mathcal{V}_{0} \oplus \mathcal{V}_{2} \oplus 2 \mathcal{V}_{4} \oplus \mathcal{V}_{6} \oplus \mathcal{V}_{8}
\end{aligned}
$$

Example 3.2. Theorem 5 implies that the invariant of degree one exist only in the case of even $d$. We can write an explicit form for these invariants. For any $d=2 m$, we consider the element

$$
I_{d}=\sum_{j+k+l=m}\left(\begin{array}{c}
m \\
j, k, l
\end{array}\right) a_{2 j, 2 k, 2 l}
$$

It is an invariant if the following conditions hold

$$
E_{1}\left(I_{d}\right)=E_{2}\left(I_{d}\right)=E_{1}\left(I_{d}\right)=0 .
$$

Let us prove that $E_{1}\left(I_{d}\right)=0$. We have

$$
E_{1}\left(I_{d}\right)=\sum_{j+k+l=d}\left(\begin{array}{c}
d \\
j, k, l
\end{array}\right) E_{1}\left(a_{2 j, 2 k, 2 l}\right)=\sum_{j+k+l=d}\left(\begin{array}{c}
d \\
j, k, l
\end{array}\right)\left(2 k a_{2 j+1,2 k-1,2 l}-2 j a_{2 j-1,2 k+1,2 l}\right) .
$$

Then

$$
\begin{aligned}
& \sum_{j+k+l=n} k\left(\begin{array}{c}
n \\
j, k, l
\end{array}\right) a_{2 j+1,2 k-1,2 l}=\sum_{\substack{j+k+l=n \\
k>0}} k\left(\begin{array}{c}
n \\
j, k, l
\end{array}\right) a_{2 j+1,2 k-1,2 l}= \\
& \sum_{\substack{j+k+l=n \\
k>0}} n\left(\begin{array}{c}
n-1 \\
j, k-1, l
\end{array}\right) a_{2 j+1,2 k-1,2 l} \stackrel{s=k-1}{=} \sum_{j+s+l=n-1} n\left(\begin{array}{c}
n-1 \\
j, s, l
\end{array}\right) a_{2 j+1,2 s+1,2 l}= \\
& \stackrel{m=j+1}{=} \sum_{\substack{m+s+l=n \\
m>0}} n\left(\begin{array}{c}
n-1 \\
m-1, s, l
\end{array}\right) a_{2 m-1,2 s+1,2 l}=\sum_{m+s+l=n} m\left(\begin{array}{c}
n \\
m, s, l
\end{array}\right) a_{2 m-1,2 s+1,2 l}= \\
& j=m \text {, } \\
& \stackrel{k=s}{=} \quad \sum_{j+k+l=n} j\left(\begin{array}{c}
n \\
j, k, l
\end{array}\right) a_{2 j-1,2 k+1,2 l} \text {. }
\end{aligned}
$$

Thus

$$
\sum_{j+k+l=n} k\left(\begin{array}{c}
n \\
j, k, l
\end{array}\right) a_{2 j+1,2 k-1,2 l}=\sum_{j+k+l=n} j\left(\begin{array}{c}
n \\
j, k, l
\end{array}\right) a_{2 j-1,2 k+1,2 l},
$$

and $E_{1}\left(I_{d}\right)=0$. In the same way, we can show that $E_{2}\left(I_{d}\right)=0$ and $E_{3}\left(I_{d}\right)=0$.

For small $d$ we have

$$
\begin{gathered}
I_{2}=a_{0,0,2}+a_{0,2,0}+a_{2,0,0} \\
I_{4}=a_{0,0,4}+2 a_{0,2,2}+a_{0,4,0}+2 a_{2,0,2}+2 a_{2,2,0}+a_{4,0,0}, \\
I_{6}=3 a_{4,0,2}+3 a_{4,2,0}+a_{6,0,0}+3 a_{0,4,2}+a_{0,6,0}+3 a_{2,0,4}+6 a_{2,2,2}+3 a_{2,4,0}+a_{0,0,6}+3 a_{0,2,4}, \\
I_{8}=6 a_{4,4,0}+4 a_{6,0,2}+4 a_{6,2,0}+a_{8,0,0}+12 a_{2,4,2}+4 a_{2,6,0}+6 a_{4,0,4}+12 a_{4,2,2}+a_{0,8,0}+4 a_{2,0,6}+ \\
+12 a_{2,2,4}+a_{0,0,8}+4 a_{0,2,6}+6 a_{0,4,4}+4 a_{0,6,2}
\end{gathered}
$$

Theorem 5 implies 
Theorem 6. The following decompositions hold:

(i) $\quad \mathbb{C}[\eta]_{d}^{S O(3)} \cong \mathbb{C}\left[l_{0}^{(d)} V_{0} \oplus l_{1}^{(d)} V_{2} \oplus l_{2}^{(d)} V_{4} \oplus \cdots \oplus l_{d}^{(d)} V_{2 d}\right]^{\mathfrak{s l}_{2}}$

(ii) $\quad \mathbb{C}(\eta)_{d}^{S O(3)} \cong \mathbb{C}\left(l_{0}^{(d)} V_{0} \oplus l_{1}^{(d)} V_{2} \oplus l_{2}^{(d)} V_{4} \oplus \cdots \oplus l_{d}^{(d)} V_{2 d}\right)^{\mathfrak{s} l_{2}}$

Therefore, it implies that the problem of determining of the algebra 3D geometric polynomial and rational moment invariants is equivalent to the problem of of determining of the algebras joint $\mathfrak{s l}_{2}$-invariants. It appears to be a very difficult problem in terms of performing calculations and it is quite a challenge to find a minimal generating set for $d>5$.

3.2. The algebra of $3 \mathrm{D}$ polynomial moment invariants $\mathbb{C}[\eta]_{2}^{S O(3)}$. Let us illustrate the above with the references to the algebra of $3 \mathrm{D}$ polynomial moment invariants of order two. Since Theorem $\left[\right.$ implies that $T_{2}^{*} \cong \mathcal{V}_{0}^{*} \oplus \mathcal{V}_{4}^{*}$, the algebra of 3D polynomial moment invariants $\mathbb{C}[\eta]_{2}^{S O(3)} \mathbb{C}[\eta]_{2}^{S O(3)}$ is equal to the algebra of $\mathfrak{s l}_{2}$-invariants $\mathbb{C}\left[\mathcal{V}_{0}\left(u_{0}\right) \oplus \mathcal{V}_{4}\left(v_{0}\right)\right]^{\mathfrak{I}_{2}}$, where $u_{0}, v_{0}$ are the lowest weight vectors in the $T_{2}^{*}$-realizations of the standard $\mathfrak{s l}_{2}$-modules $\mathcal{V}_{0}$ and $\mathcal{V}_{4}$.

To find such a realization, firstly we need to find the realization of the standard basis of $\mathcal{V}_{0}$ and $\mathcal{V}_{4}$ on $T_{2}^{*}$ and, then, substitute it into the expressions for the generating invariants of the algebra $\mathbb{C}\left[\mathcal{V}_{0}\left(u_{0}\right) \oplus \mathcal{V}_{4}\left(v_{0}\right)\right]^{\mathfrak{s l}_{2}}$. Since $\mathcal{V}_{0}$ is a trivial $\mathfrak{s l}_{2}$-module, it is enough to find the generating elements of the alebra $\mathbb{C}\left[V_{4}\left(v_{0}\right)\right]^{\mathfrak{s l}_{2}}$. But $\mathbb{C}\left[V_{4}\left(v_{0}\right)\right]^{\mathfrak{s l}_{2}}$ which is isomorphic to the classical algebra of invariants of binary form of degree four

$$
a_{0} x^{4}+4 a_{1} x^{3} y+6 a_{2} x^{2} y^{2}+4 a_{3} x y^{3}+a_{4} y^{4} .
$$

It is well-known, that the latter is generated by the following two invariants of degree two and three:

$$
\begin{aligned}
& S_{1}=a_{0} a_{4}+3 a_{2}^{2}-4 a_{1} a_{3}, \\
& S_{2}=a_{0} a_{2} a_{4}+2 a_{1} a_{2} a_{3}-a_{2}^{3}-a_{0} a_{3}^{2}-a_{1}^{2} a_{4}=\left|\begin{array}{lll}
a_{0} & a_{1} & a_{2} \\
a_{1} & a_{2} & a_{3} \\
a_{2} & a_{3} & a_{4}
\end{array}\right| .
\end{aligned}
$$

In terms of the classical invariant theory, the invariant $S_{1}$ is called the apolar invariant and the invariant $S_{2}$ is known as the the catalecticant or the Hankel determinant.

The six-dimensional $s l_{2}$-module $T_{2}^{*}$ is generated by the following elements:

$$
T_{2}^{*}=\left\langle a_{0,0,2}, a_{0,1,1}, a_{0,2,0}, a_{1,0,1}, a_{1,1,0}, a_{2,0,0}\right\rangle .
$$

The operators $\mathcal{D}_{+}, \mathcal{D}_{-}, \mathcal{H}$ act on the basis as follows (see Theorem 11):

$$
\begin{aligned}
& \mathcal{D}_{+}\left(a_{j, k, l}\right)=i\left(k a_{j+1, k-1, l}-j a_{j-1, k+1, l}\right)+l a_{j+1, k, l-1}-j a_{j-1, k, l+1}, \\
& \mathcal{D}_{-}\left(a_{j, k, l}\right)=i\left(k a_{j+1, k-1, l}-j a_{j-1, k+1, l}\right)-\left(l a_{j+1, k, l-1}-j a_{j-1, k, l+1}\right), \\
& \mathcal{H}\left(a_{j, k, l}\right)=2 i\left(l a_{j, k+1, l-1}-k a_{j, k-1, l+1}\right) .
\end{aligned}
$$

The lowest weight vectors $u_{0}, v_{0}$ of the $\mathfrak{s l}_{2}$-modules $\mathcal{V}_{0}\left(u_{0}\right)$ and $\mathcal{V}_{4}\left(v_{0}\right)$ are the solutions of the following two systems of linear equations: $\left\{\begin{array}{l}\mathcal{E}_{-}(z)=0, \\ \mathcal{H}(z)=0\end{array}\right.$ and $\left\{\begin{array}{l}\mathcal{E}_{-}(z)=0, \\ \mathcal{H}(z)=4 z\end{array}\right.$, respectively. Thus, we obtain

$$
\begin{aligned}
& u_{0}=I_{1}=a_{0,0,2}+a_{0,2,0}+a_{2,0,0}, \\
& v_{0}=2 a_{0,1,1}+i\left(a_{0,0,2}-a_{0,2,0}\right) .
\end{aligned}
$$

The element $u_{0}$ is already an invariant. 
Using Theorem 4, we get the standard basis $\mathcal{V}_{4}\left(v_{0}\right)$ :

$$
\begin{aligned}
& v_{0}=v_{0}=2 a_{0,1,1}+i\left(a_{0,0,2}-a_{0,2,0}\right), \\
& v_{1}=\frac{1}{4} D_{+}\left(x_{0}\right)=i a_{1,0,1}+a_{1,1,0}, \\
& v_{2}=\frac{1}{12} D_{+}^{2}\left(x_{0}\right)=-\frac{i}{3}\left(a_{0,0,2}+a_{0,2,0}-2 a_{2,0,0}\right), \\
& v_{3}=\frac{1}{24} D_{+}^{3}\left(x_{0}\right)=a_{1,1,0}-i a_{1,0,1}, \\
& v_{4}=\frac{1}{24} D_{+}^{4}\left(x_{0}\right)=-2 a_{0,1,1}+i\left(-a_{0,2,0}+a_{0,0,2}\right)
\end{aligned}
$$

Substituting $v_{i}$ for $a_{i}$ in $S_{1}, S_{2}$ we find invariants $I_{2}$ and $I_{3}$ :

$$
\begin{aligned}
I_{2}= & a_{0,0,2}{ }^{2}-a_{0,0,2} a_{0,2,0}-a_{0,0,2} a_{2,0,0}+3{a_{0,1,1}}^{2}+a_{0,2,0}{ }^{2}-a_{0,2,0} a_{2,0,0}+3 a_{1,0,1}{ }^{2}+3 a_{1,1,0}{ }^{2}+a_{2,0,0}{ }^{2}, \\
I_{3}= & 2 a_{0,0,2}{ }^{3}-3 a_{0,0,2}{ }^{2} a_{2,0,0}+9 a_{0,0,2} a_{0,1,1}{ }^{2}-3 a_{0,2,0}{ }^{2} a_{0,0,2}+12 a_{0,0,2} a_{2,0,0} a_{0,2,0}+ \\
& +9 a_{0,0,2} a_{1,0,1}{ }^{2}-18 a_{0,0,2} a_{1,1,0}{ }^{2}-3 a_{0,0,2} a_{2,0,0}{ }^{2}+9 a_{0,1,1}{ }^{2} a_{0,2,0}-18 a_{0,1,1}{ }^{2} a_{2,0,0}+ \\
& +54 a_{0,1,1} a_{1,1,0} a_{1,0,1}-3 a_{0,2,0}{ }^{2} a_{2,0,0}-18 a_{0,2,0} a_{1,0,1}{ }^{2}+9 a_{0,2,0} a_{1,1,0}{ }^{2}-3 a_{0,2,0} a_{2,0,0}{ }^{2}+ \\
& +9 a_{1,0,1}{ }^{2} a_{2,0,0}+9 a_{1,1,0}{ }^{2} a_{2,0,0}+2 a_{2,0,0}{ }^{3}-3 a_{0,0,2}{ }^{2} a_{0,2,0}+2 a_{0,2,0} .
\end{aligned}
$$

Thus, we have proved the following theorem.

Theorem 7. The algebras of polynomial and rational invariants $\mathbb{C}\left[T_{2}\right]^{\mathfrak{s} \mathfrak{I}_{2}}, \mathbb{C}\left(T_{2}\right)^{\mathfrak{s l}_{2}}$ are generated by the invariants $I_{1}, I_{2}$ and $I_{3}$ :

$$
\begin{aligned}
& \mathbb{C}\left[T_{2}\right]^{s l_{2}}=\mathbb{C}\left[I_{1}, I_{2}, I_{3}\right], \\
& \mathbb{C}\left(T_{2}\right)^{s l_{2}}=\mathbb{C}\left(I_{1}, I_{2}, I_{3}\right) .
\end{aligned}
$$

In order to obtain the 3D moment invariant it is sufficient to replace $a_{j, k, l}$ by the normalized moments $\eta_{j, k, l}$ in $I_{1}, I_{2}$ and $I_{3}$.

We should admit that the obtained result is confirmed by the result of [5], 6], 8] obtained by a different method.

As far as the obtained expressions for the invariants are quite cumbersome, we are interested in finding a simpler representation for them. Let us consider the Laplace operator:

$$
\mathcal{L}=\mathcal{D}_{+} \mathcal{D}_{-}+\mathcal{D}_{-} \mathcal{D}_{+}+\frac{1}{2} \mathcal{H}^{2}=E_{1}^{2}+E_{2}^{2}+E_{3}^{2},
$$

which belongs to the enveloping algebra of $\mathfrak{s l}_{2}$. It can be proved that $\mathcal{L}$ commutes with the operators $\mathcal{D}_{+}, \mathcal{D}_{-}, \mathcal{H}$. Therefore, $\mathcal{L}$ is diagonalizable on every standard $\mathfrak{s l}_{2}$-module.

Let us express the invariants in terms of the eigenvectors of the Laplace operator $\mathcal{L}$. The operator $\mathcal{L}$ acts on the basis of $T_{2}^{*}$ as follows:

$$
\begin{aligned}
& \mathcal{L}\left(a_{0,0,2}\right)=-4 a_{2,0,0}+8 a_{0,0,2}-4 a_{0,2,0}, \mathcal{L}\left(a_{0,1,1}\right)=12 a_{0,1,1}, \\
& \mathcal{L}\left(a_{0,2,0}\right)=8 a_{0,2,0}-4 a_{2,0,0}-4 a_{0,0,2}, \mathcal{L}\left(a_{1,0,1}\right)=12 a_{1,0,1}, \\
& \mathcal{L}\left(a_{1,1,0}\right)=12 a_{1,1,0}, \mathcal{L}\left(a_{2,0,0)}=-4 a_{0,2,0}+8 a_{2,0,0}-4 a_{0,0,2} .\right.
\end{aligned}
$$

Since $T_{2}^{*}=\mathcal{V}_{0}\left(u_{0}\right) \oplus \mathcal{V}_{4}\left(v_{0}\right)$, then there exists one eigenvector, let us denote it by $e_{0}$, associated with the zero eigevalue and five eigenvectors $e_{1}, e_{2}, e_{3}, e_{4}, e_{5}$ associated with the eigevalue 
twelve. The eigenvectors could be found by the standard linear algebra algorithm:

$$
\begin{aligned}
& e_{0}=a_{0,0,2}+a_{0,2,0}+a_{2,0,0} \\
& e_{1}=a_{0,1,1}, e_{2}=a_{0,2,0}-a_{0,0,2}, e_{3}=a_{1,0,1}, e_{4}=a_{1,1,0}, e_{5}=a_{2,0,0}-a_{0,0,2} .
\end{aligned}
$$

Then the invariants $I_{1}, I_{2}$ and $I_{3}$ are expressed in a much more compact form:

$$
\begin{aligned}
& I_{1}=e_{0}, \\
& I_{2}=3 e_{1}^{2}+e_{2}^{2}-e_{5} e_{2}+3 e_{3}^{2}+3 e_{4}^{2}+e_{5}^{2}, \\
& I_{3}=9 e_{1}^{2} e_{2}-18 e_{1}^{2} e_{5}+54 e_{1} e_{4} e_{3}+2 e_{2}^{3}-3 e_{5} e_{2}^{2}-18 e_{3}^{2} e_{2}+9 e_{4}^{2} e_{2}-3 e_{5}^{2} e_{2}+9 e_{3}^{2} e_{5}+9 e_{4}^{2} e_{5}+2 e_{5}^{3} .
\end{aligned}
$$

3.3. The algebra of polynomial invariants $\mathbb{C}\left[U_{3}\right]^{\mathfrak{s l}_{2}}$. We note, that the case $d=3$ is much more complicated than the case $d=2$. For $d=3$ we have the following decomposition of the $\mathfrak{s l}_{2}$-module $U_{3}$ :

$$
U_{3}=T_{2}^{*} \oplus T_{3}^{*} \cong \mathcal{V}_{0}\left(v_{0}\right) \oplus \mathcal{V}_{2}\left(x_{0}\right) \oplus \mathcal{V}_{4}\left(y_{0}\right) \oplus \mathcal{V}_{6}\left(u_{0}\right)
$$

Suppose the $\mathfrak{s l}_{2}$-modules $\mathcal{V}_{0}, \mathcal{V}_{2}, \mathcal{V}_{4}, \mathcal{V}_{6}$ are given by their standard bases:

$$
\begin{aligned}
& \mathcal{V}_{0}\left(v_{0}\right)=\left\langle v_{0}\right\rangle \\
& \mathcal{V}_{2}\left(x_{0}\right)=\left\langle x_{0}, x_{1}, x_{2}\right\rangle \\
& \mathcal{V}_{4}\left(y_{0}\right)=\left\langle y_{0}, y_{1}, y_{2}, y_{3}, y_{4}\right\rangle \\
& \mathcal{V}_{6}\left(u_{0}\right)=\left\langle u_{0}, u_{1}, u_{2}, u_{3}, u_{4}, u_{5}, u_{6}\right\rangle .
\end{aligned}
$$

Proceeding as above, we again find the lowest weight vectors $v_{0}, x_{0}, y_{0}, u_{0}$ by solving systems of linear equations. Further, by using Theorem 4 we obtain the following realization of all these $\mathfrak{s l}_{2}$-modules in $U_{3}$ :

$$
\begin{aligned}
& v_{0}=a_{0,0,2}+a_{0,2,0}+a_{2,0,0}, \\
& x_{0}=a_{0,0,3}+a_{0,2,1}+a_{2,0,1}-i\left(a_{0,1,2}+a_{0,3,0}+a_{2,1,0}\right), x_{1}=a_{1,0,2}+a_{1,2,0}+a_{3,0,0}, x_{2}=-\bar{x}_{0}, \\
& y_{0}=2 a_{0,1,1}+i\left(a_{0,0,2}-a_{0,2,0}\right), y_{1}=a_{1,1,0}+i a_{1,0,1}, y_{2}=\frac{i}{3}\left(2 a_{2,0,0}-a_{0,0,2}-a_{0,2,0}\right), y_{3}=\bar{y}_{1}, y_{4}=-\bar{y}_{0}, \\
& u_{0}=a_{0,0,3}-3 a_{0,2,1}+i\left(a_{0,3,0}-3 a_{0,1,2}\right), u_{1}=a_{1,0,2}-a_{1,2,0}-2 i a_{1,1,1}, \\
& u_{2}=\frac{1}{5}\left(4 a_{2,0,1}-a_{0,0,3}-a_{0,2,1}+i\left(a_{0,1,2}+a_{0,3,0}-4 a_{2,1,0}\right)\right), \\
& u_{3}=\frac{1}{5}\left(2 a_{3,0,0}-3 a_{1,0,2}-3 a_{1,2,0}\right), u_{4}=-\bar{u}_{2}, u_{5}=\bar{u}_{1}, u_{6}=-\bar{u}_{0} .
\end{aligned}
$$

Here - indicates the complex conjugate.

Recently, the minimal generating set of polynomial invariants for the algebra $\mathbb{C}\left[\mathcal{V}_{2} \oplus \mathcal{V}_{4} \oplus\right.$ $\left.\mathcal{V}_{6}\right]^{\mathfrak{s l}_{2}}$ was calculated, see [13], in the symbolic form. The minimal generating set consists of 195 invariants and their degrees grow up to fifteen. Therefore, a minimal generating set of polynomial invariants of the algebra $\mathbb{C}\left[T_{3}\right]^{\mathfrak{s l}_{2}}$ consists of 196 invariants. These invariants can be calculated explicitly using author's Maple package [16] or by expanding the transvectants listed in the paper [13]. Below we present only the first thirteen invariants: 


\begin{tabular}{|c|c|c|}
\hline deg & Invariants & \# \\
\hline 1 & $v_{0}$ & 1 \\
\hline 2 & $x_{0} x_{2}-x_{1}^{2}, y_{0} y_{4}-4 y_{1} y_{3}+3 y_{2}^{2}, u_{0} u_{6}-6 u_{1} u_{5}+15 u_{2} u_{4}-10 u_{3}^{2}$ & 3 \\
\hline 3 & $\begin{array}{l}y_{0} y_{2} y_{4}-y_{0} y_{3}^{2}-y_{1}^{2} y_{4}+2 y_{1} y_{2} y_{3}-y_{2}^{3} \\
x_{0}^{2} y_{4}-4 x_{0} x_{1} y_{3}+2 x_{0} x_{2} y_{2}+4 x_{1}^{2} y_{2}-4 x_{1} x_{2} y_{1}+x_{2}^{2} y_{0} \\
u_{0} u_{4} y_{4}-2 u_{0} u_{5} y_{3}-4 u_{1} u_{3} y_{4}+6 u_{1} u_{4} y_{3}-2 u_{1} u_{6} y_{1}+3 u_{2}^{2} y_{4}-4 u_{2} u_{3} y_{3}- \\
-9 u_{2} u_{4} y_{2}+6 u_{2} u_{5} y_{1}+u_{2} u_{6} y_{0}+8 u_{3}^{2} y_{2}-4 u_{3} u_{4} y_{1}-4 u_{3} u_{5} y_{0}+3 u_{4}^{2} y_{0}+u_{0} u_{6} y_{2}, \\
u_{0} x_{2} y_{4}-2 u_{1} x_{1} y_{4}-4 u_{1} x_{2} y_{3}+8 u_{2} x_{1} y_{3}+6 u_{2} x_{2} y_{2}-4 u_{3} x_{0} y_{3}-12 u_{3} x_{1} y_{2}- \\
-4 u_{3} x_{2} y_{1}+6 u_{4} x_{0} y_{2}+8 u_{4} x_{1} y_{1}+u_{4} x_{2} y_{0}-4 u_{5} x_{0} y_{1}-2 u_{5} x_{1} y_{0}+u_{6} x_{0} y_{0}+u_{2} x_{0} y_{4}\end{array}$ & 4 \\
\hline 4 & 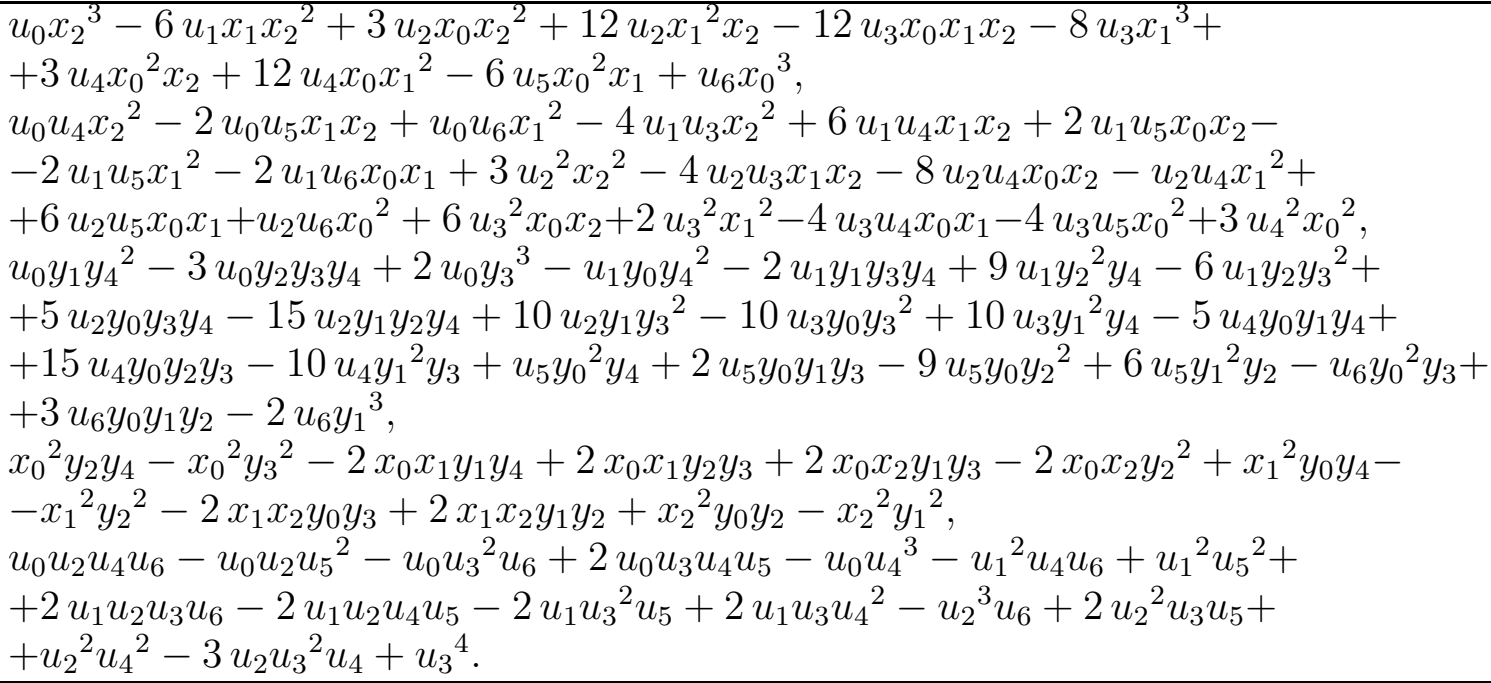 & 5 \\
\hline
\end{tabular}

Substituting the realizations of the standard $\mathfrak{s l}_{2}$-modules in the invariants expressions, we get the explicit expressions for the invariants of the algebra $\mathbb{C}\left[T_{3}\right]^{\mathfrak{s l}_{2}}$. In order to obtain the 3D moment invariant it is sufficient to replace $a_{j, k, l}$ by the normalized moments $\eta_{j, k, l}$. For example, the 3D geometric moment invariants of low degrees have the form

$$
\begin{aligned}
& B_{0}=\eta_{0,0,2}+\eta_{0,2,0}+\eta_{2,0,0}, \\
& B_{1}=\eta_{0,0,2}{ }^{2}-\eta_{0,0,2} \eta_{0,2,0}-\eta_{0,0,2} \eta_{2,0,0}+3 \eta_{0,1,1}{ }^{2}+\eta_{0,2,0}{ }^{2}-\eta_{0,2,0} \eta_{2,0,0}+3 \eta_{1,0,1}{ }^{2}+3 \eta_{1,1,0}{ }^{2}+\eta_{2,0,0}{ }^{2} \text {, } \\
& B_{2}=\eta_{0,0,3}{ }^{2}+2 \eta_{0,0,3} \eta_{0,2,1}+2 \eta_{0,0,3} \eta_{2,0,1}+\eta_{0,1,2}^{2}+2 \eta_{0,1,2} \eta_{0,3,0}+2 \eta_{0,1,2} \eta_{2,1,0}+\eta_{0,2,1}{ }^{2}+ \\
& +2 \eta_{0,2,1} \eta_{2,0,1}+\eta_{0,3,0}{ }^{2}+2 \eta_{0,3,0} \eta_{2,1,0}+\eta_{1,0,2}^{2}+2 \eta_{1,0,2} \eta_{1,2,0}+2 \eta_{1,0,2} \eta_{3,0,0}+\eta_{1,2,0}{ }^{2}+ \\
& +2 \eta_{1,2,0} \eta_{3,0,0}+\eta_{2,0,1}^{2}+\eta_{2,1,0}^{2}+\eta_{3,0,0}^{2}, \\
& B_{3}=\eta_{0,0,3}{ }^{2}-3 \eta_{0,0,3} \eta_{0,2,1}-3 \eta_{0,0,3} \eta_{2,0,1}+6 \eta_{0,1,2}{ }^{2}-3 \eta_{0,1,2} \eta_{0,3,0}-3 \eta_{0,1,2} \eta_{2,1,0}+6 \eta_{0,2,1}{ }^{2}- \\
& -3 \eta_{0,2,1} \eta_{2,0,1}+\eta_{0,3,0}{ }^{2}-3 \eta_{0,3,0} \eta_{2,1,0}+6 \eta_{1,0,2}{ }^{2}-3 \eta_{1,0,2} \eta_{1,2,0}-3 \eta_{1,0,2} \eta_{3,0,0}+15 \eta_{1,1,1}{ }^{2}+ \\
& +6 \eta_{1,2,0}^{2}-3 \eta_{1,2,0} \eta_{3,0,0}+6{\eta_{2,0,1}}^{2}+6 \eta_{2,1,0}^{2}+\eta_{3,0,0}^{2} \text {. }
\end{aligned}
$$

All of the 196 invariants can be obtained in a similar way as above.

In the book [8], the 3D moment invariants $\Phi_{1}, \ldots, \Phi_{13}$ were presented, in particular, the first degree invariant $\Phi_{1}$ and the invariants $\Phi_{2}, \Phi_{4}, \Phi_{5}$ of degree two. These invariants could be expressed in terms of the invariants $B_{0}, B_{1}, B_{2}, B_{3}$ as follows:

$$
\Psi_{1}=B_{0}, \Phi_{2}=\frac{B_{0}^{2}+2 B_{1}}{3}, \Phi_{4}=\frac{3 B_{2}+2 B_{3}}{5}, \Phi_{5}=B_{2} .
$$


The Poincaré series of the algebra $\mathbb{C}\left[T_{3}\right]^{\mathfrak{s l}_{2}}$ calculated by using Maple package (see [17]) has the form:

$$
\begin{gathered}
\mathcal{P}\left(\mathbb{C}\left[T_{3}\right]^{s l_{2}}, z\right)=\frac{p_{0246}(z)}{(1-z)\left(1-z^{6}\right)\left(1-z^{5}\right)^{2}\left(1-z^{4}\right)^{3}\left(1-z^{3}\right)^{3}\left(1-z^{2}\right)^{3}}= \\
=1+z+4 z^{2}+8 z^{3}+26 z^{4}+53 z^{5}+146 z^{6}+305 z^{7}+704 z^{8}+1417 z^{9}+\cdots
\end{gathered}
$$

where

$$
\begin{gathered}
p_{0246}(z)=z^{28}+z^{25}+9 z^{24}+13 z^{23}+37 z^{22}+51 z^{21}+91 z^{20}+119 z^{19}+181 z^{18}+208 z^{17}+ \\
+277 z^{16}+283 z^{15}+311 z^{14}+283 z^{13}+277 z^{12}+208 z^{11}+181 z^{10}+119 z^{9}+91 z^{8}+51 z^{7}+ \\
+37 z^{6}+13 z^{5}+9 z^{4}+z^{3}+1
\end{gathered}
$$

Therefore, the algebra $\mathbb{C}[\eta]_{3}^{\mathfrak{s l}_{2}}$ consists of one invariant of degree 1 , namely $B_{0}$. Also, there exists four linearly independent invariants of degree two, namely $B_{0}^{2}, B_{1}, B_{2}, B_{3}$, eight linearly independent invariants of degree three etc.

\section{The algebra of Rational invariants $\mathbb{C}\left(U_{d}\right)^{\mathfrak{s l}_{2}}$.}

Concidering applications, the rational invariants are more interesting applications than the polynomial ones. In the paper [9], a set of 1185 of the 3D rotational moment invariants up to the sixteenth order was presented. However, these invariants do not form a minimal generating system and setting a minimal generating system is still remaining an open problem.

In the following theorem we find the cardinality of a minimal generating set of the algebra of $3 \mathrm{D}$ rational rotation invariants.

Theorem 8. The number of elements in a minimal generating set of the algebra of the rational invariants $\mathbb{C}\left(U_{d}\right)^{\mathfrak{s l}_{2}}, d \geq 2$ is equal to

$$
\left(\begin{array}{c}
d+3 \\
3
\end{array}\right)-7
$$

Proof. Since the group $S L(2)$ as an affine variety is three-dimensional one, then, the transcendence degree of the field extension $\mathbb{C}\left(U_{d}\right)^{S O(3)} / \mathbb{C}$ equals to

$$
\operatorname{tr} \operatorname{deg}_{\mathbb{C}} \mathbb{C}\left(U_{d}\right)^{S O(3)}=\operatorname{dim} U_{d}-\operatorname{dim} S O(3) .
$$

Thus, the algebra $\mathbb{C}\left(U_{d}\right)^{\mathfrak{s l}_{2}}$ consists of exactly $\operatorname{dim} U_{d}-3$ algebraically independent elements. Taking into account that

$$
\operatorname{dim} U_{d}-3=\sum_{k=2}^{d} \operatorname{dim} T_{d}-3=\sum_{k=2}^{d}\left(\begin{array}{c}
k+2 \\
2
\end{array}\right)-3=\left(\begin{array}{c}
d+3 \\
3
\end{array}\right)-7
$$

which is equal to that to be proved.

In particular, for $d=2,3$ we have three and thirteen invariants, respectively. These results are confirmed by the results in [8]. For $d=2$, it implies that the algebra $\mathbb{C}\left(U_{2}\right)^{\mathfrak{s l}_{2}}$ is generated by the invariants $I_{1}, I_{2}$ and $I_{3}$.

A system of 13 invariants of the algebra $\mathbb{C}\left(U_{3}\right)^{\mathfrak{s l}_{2}}$ was presented in [8].

The authors claim, without proof, that these invariants are independent. Below we present another system of thirteen invariants for $\mathbb{C}\left(U_{3}\right)^{\mathfrak{s l}_{2}}$ and prove that all these invariants form a minimal generating set of the algebra rational invariants $\mathbb{C}\left(U_{3}\right)^{\mathfrak{s l}_{2}}$.

In the Sect. 3.3 we found an explicit form for each of the thirteen polynomial invariants of the algebra $\mathbb{C}\left[U_{3}\right]^{\mathfrak{s l}_{2}}$.Though, the expressions for the invariants are quite cumbersome, we will 
express them in terms of the eigenvectors of the Laplace operator $\mathcal{L}$. The operator $\mathcal{L}$ acts on the basis of $T_{3}^{*}$ as follows:

$$
\begin{aligned}
& \mathcal{L}\left(a_{0,0,3}\right)=-12 a_{2,0,1}+12 a_{0,0,3}-12 a_{0,2,1}, \mathcal{L}\left(a_{0,1,2}\right)=20 a_{0,1,2}-4 a_{2,1,0}-4 a_{0,3,0} \\
& \mathcal{L}\left(a_{0,2,1}\right)=20 a_{0,2,1}-4 a_{2,0,1}-4 a_{0,0,3}, \mathcal{L}\left(a_{0,3,0}\right)=-12 a_{2,1,0}+12 a_{0,3,0}-12 a_{0,1,2} \\
& \mathcal{L}\left(a_{1,0,2}\right)=20 a_{1,0,2}-4 a_{3,0,0}-4 a_{1,2,0}, \mathcal{L}\left(a_{1,1,1}\right)=24 a_{1,1,1} \\
& \mathcal{L}\left(a_{1,2,0}\right)=20 a_{1,2,0}-4 a_{3,0,0}-4 a_{1,0,2}, \mathcal{L}\left(a_{2,0,1}\right)=-4 a_{0,2,1}+20 a_{2,0,1}-4 a_{0,0,3} \\
& \mathcal{L}\left(a_{2,1,0}\right)=-4 a_{0,3,0}+20 a_{2,1,0}-4 a_{0,1,2}, \mathcal{L}\left(a_{3,0,0}\right)=-12 a_{1,2,0}+12 a_{3,0,0}-12 a_{1,0,2}
\end{aligned}
$$

Let us recall that $T_{3}^{*}=\mathcal{V}_{2}\left(y_{0}\right) \oplus \mathcal{V}_{6}\left(u_{0}\right)$. Let $c_{1}, c_{2}, c_{3}$ and $b_{1}, b_{2}, b_{3}, b_{4}, b_{4}, b_{5}, b_{7}$ denote the eigenvectors of $\mathcal{L}$ in the vector spaces $\mathcal{V}_{2}\left(y_{0}\right)$ and $\mathcal{V}_{6}\left(u_{0}\right)$, respectively. We find the eigenvectors by the standard linear algebra algorithm:

$$
\begin{aligned}
& c_{1}=a_{0,0,3}+a_{0,2,1}+a_{2,0,1}, c_{2}=a_{0,1,2}+a_{0,3,0}+a_{2,1,0}, c_{3}=a_{1,0,2}+a_{1,2,0}+a_{3,0,0} \\
& b_{1}=a_{0,0,3}-3 a_{0,2,1}, b_{2}=-3 a_{0,1,2}+a_{0,3,0}, b_{3}=a_{1,1,1} \\
& b_{4}=a_{1,2,0}-a_{1,0,2}, b_{5}=a_{2,0,1}-a_{0,2,1}, b_{6}=a_{2,1,0}-a_{0,1,2}, b_{7}=-3 a_{1,0,2}+a_{3,0,0} .
\end{aligned}
$$

The eigenvectors for the spaces $\mathcal{V}_{0}\left(u_{0}\right)$ and $\mathcal{V}_{4}\left(x_{0}\right)$ we already found in Subsect. 3.2. Now, let us express the above thirteen invariants in terms of the eigenvectors. We have

$$
\begin{aligned}
& \boldsymbol{o d}=e_{0}, \\
& \boldsymbol{d} \boldsymbol{v}_{1}=c_{1}^{2}+c_{2}^{2}+c_{3}^{2} \\
& \boldsymbol{d} \boldsymbol{v}_{2}=3 e_{1}{ }^{2}+e_{2}{ }^{2}-e_{5} e_{2}+3 e_{3}{ }^{2}+3 e_{4}{ }^{2}+e_{5}{ }^{2}, \\
& \boldsymbol{d} \boldsymbol{v}_{3}=b_{1}{ }^{2}-3 b_{5} b_{1}+b_{2}{ }^{2}-3 b_{6} b_{2}+15 b_{3}{ }^{2}+6 b_{4}{ }^{2}-3 b_{4} b_{7}+6 b_{5}{ }^{2}+6 b_{6}{ }^{2}+b_{7}{ }^{2}, \\
& \boldsymbol{t r}_{1}=9 e_{1}^{2} e_{2}-18 e_{1}^{2} e_{5}+54 e_{1} e_{4} e_{3}+2 e_{2}^{3}-3 e_{5} e_{2}^{2}-18 e_{3}^{2} e_{2}+9 e_{4}^{2} e_{2}-3 e_{5}^{2} e_{2}+9 e_{3}^{2} e_{5}+ \\
& +9 e_{4}^{2} e_{5}+2 e_{5}^{3}, \\
& \boldsymbol{t} \boldsymbol{r}_{2}=c_{1}^{2} e_{2}+c_{1}^{2} e_{5}-6 e_{1} c_{1} c_{2}-6 e_{3} c_{3} c_{1}-2 c_{2}^{2} e_{2}+c_{2}^{2} e_{5}-6 e_{4} c_{3} c_{2}+c_{3}^{2} e_{2}-2 c_{3}^{2} e_{5}, \\
& \boldsymbol{t r}_{3}=2 b_{1}^{2} e_{2}+2 b_{1}^{2} e_{5}+3 e_{1} b_{2} b_{1}+60 e_{4} b_{3} b_{1}+3 e_{3} b_{1} b_{4}-21 b_{1} b_{5} e_{2}+9 b_{1} b_{5} e_{5}+ \\
& +3 e_{1} b_{6} b_{1}+3 e_{3} b_{1} b_{7}-4 b_{2}^{2} e_{2}+2 b_{2}^{2} e_{5}+60 b_{2} b_{3} e_{3}-12 e_{4} b_{2} b_{4}+3 e_{1} b_{2} b_{5}+12 b_{2} b_{6} e_{2}+ \\
& +9 b_{2} b_{6} e_{5}+3 e_{4} b_{2} b_{7}-90 b_{3} e_{1} b_{4}-90 e_{4} b_{3} b_{5}-90 b_{3} b_{6} e_{3}+60 b_{3} e_{1} b_{7}-18 b_{4}{ }^{2} e_{2}-9 b_{4}{ }^{2} e_{5}+ \\
& +63 e_{3} b_{5} b_{4}-27 e_{4} b_{6} b_{4}+9 b_{4} b_{7} e_{2}+12 b_{4} b_{7} e_{5}+27 b_{5}{ }^{2} e_{2}-18 b_{5}{ }^{2} e_{5}-72 e_{1} b_{6} b_{5}-12 e_{3} b_{5} b_{7}- \\
& -9 b_{6}{ }^{2} e_{2}-18 b_{6}^{2} e_{5}-12 e_{4} b_{6} b_{7}+2 b_{7}^{2} e_{2}-4 b_{7}^{2} e_{5}, \\
& \boldsymbol{t r}_{4}=c_{1} b_{1} e_{2}+c_{1} b_{1} e_{5}+2 e_{1} c_{2} b_{1}+2 e_{3} c_{3} b_{1}+2 e_{1} c_{1} b_{2}-2 c_{2} b_{2} e_{2}+c_{2} b_{2} e_{5}+2 e_{4} c_{3} b_{2}- \\
& -10 e_{4} b_{3} c_{1}-10 b_{3} c_{2} e_{3}-10 b_{3} e_{1} c_{3}+2 e_{3} c_{1} b_{4}-8 e_{4} c_{2} b_{4}-4 b_{4} c_{3} e_{2}+3 b_{4} c_{3} e_{5}+b_{5} c_{1} e_{2}- \\
& -4 b_{5} c_{1} e_{5}+2 e_{1} c_{2} b_{5}-8 e_{3} c_{3} b_{5}+2 e_{1} c_{1} b_{6}+3 b_{6} c_{2} e_{2}-4 b_{6} c_{2} e_{5}- \\
& -8 e_{4} c_{3} b_{6}+2 e_{3} c_{1} b_{7}+2 e_{4} c_{2} b_{7}+c_{3} b_{7} e_{2}-2 c_{3} b_{7} e_{5} \\
& \boldsymbol{c h}_{1}=2 b_{1} c_{1}^{3}-3 c_{2}^{2} c_{1} b_{1}-3 b_{1} c_{3}{ }^{2} c_{1}-3 c_{2} b_{2} c_{1}{ }^{2}+2 b_{2} c_{2}^{3}-3 b_{2} c_{3}^{2} c_{2}+30 b_{3} c_{2} c_{3} c_{1}- \\
& -3 c_{1}{ }^{2} b_{4} c_{3}+12 c_{2}{ }^{2} b_{4} c_{3}-3 b_{4} c_{3}{ }^{3}-3 b_{5} c_{1}{ }^{3}-3 c_{2}{ }^{2} c_{1} b_{5}+12 b_{5} c_{3}{ }^{2} c_{1}-3 b_{6} c_{2} c_{1}{ }^{2}-3 b_{6} c_{2}{ }^{3}+ \\
& +12 b_{6} c_{3}^{2} c_{2}-3 c_{1}^{2} c_{3} b_{7}-3 c_{2}^{2} c_{3} b_{7}+2 c_{3}^{3} b_{7},
\end{aligned}
$$




$$
\begin{gathered}
\boldsymbol{c h}_{2}=b_{1}{ }^{2} c_{1}{ }^{2}-3{c_{2}}^{2} b_{1}{ }^{2}-3 b_{1}{ }^{2} c_{3}{ }^{2}-2 c_{2} b_{2} c_{1} b_{1}-40 b_{3} c_{2} c_{3} b_{1}-2 b_{1} b_{4} c_{3} c_{1}-3 b_{1} b_{5} c_{1}{ }^{2}+ \\
+19 c_{2}{ }^{2} b_{1} b_{5}-b_{1} c_{3}{ }^{2} b_{5}-2 b_{6} c_{2} c_{1} b_{1}-2 b_{1} c_{3} b_{7} c_{1}-3 b_{2}{ }^{2} c_{1}{ }^{2}+b_{2}{ }^{2} c_{2}{ }^{2}-3 b_{2}{ }^{2} c_{3}{ }^{2}-40 b_{3} b_{2} c_{3} c_{1}+ \\
+8 b_{2} b_{4} c_{3} c_{2}-2 c_{2} b_{2} c_{1} b_{5}+19 b_{6} b_{2} c_{1}{ }^{2}-3 b_{2} b_{6} c_{2}{ }^{2}-b_{2} c_{3}{ }^{2} b_{6}-2 b_{2} c_{3} b_{7} c_{2}-25 b_{3}{ }^{2} c_{1}{ }^{2}- \\
-25 b_{3}{ }^{2} c_{2}{ }^{2}-25 b_{3}{ }^{2} c_{3}{ }^{2}+60 b_{3} c_{2} b_{4} c_{1}+60 b_{3} c_{2} c_{3} b_{5}+60 b_{3} b_{6} c_{3} c_{1}-40 b_{3} c_{2} b_{7} c_{1}-28 b_{4}{ }^{2} c_{1}{ }^{2}+ \\
+2 c_{2}{ }^{2} b_{4}{ }^{2}-4 b_{4}{ }^{2} c_{3}{ }^{2}-42 b_{5} b_{4} c_{3} c_{1}+18 b_{6} b_{4} c_{3} c_{2}+19 b_{4} b_{7} c_{1}{ }^{2}-c_{2}{ }^{2} b_{4} b_{7}-3 b_{4} c_{3}{ }^{2} b_{7}-4 b_{5}{ }^{2} c_{1}{ }^{2}- \\
-28 c_{2}{ }^{2} b_{5}{ }^{2}+2 b_{5}{ }^{2} c_{3}{ }^{2}+48 b_{6} c_{2} b_{5} c_{1}+8 b_{5} c_{3} b_{7} c_{1}-28 b_{6}{ }^{2} c_{1}{ }^{2}-4 b_{6}{ }^{2} c_{2}{ }^{2}+2 b_{6}{ }^{2} c_{3}{ }^{2}+ \\
+8 b_{6} c_{3} b_{7} c_{2}-3 b_{7}{ }^{2} c_{1}{ }^{2}-3 c_{2}{ }^{2} b_{7}{ }^{2}+c_{3}{ }^{2} b_{7}{ }^{2},
\end{gathered}
$$

$$
\begin{aligned}
c h_{3}= & b_{1} e_{1}^{2} e_{4}-b_{1} e_{1} e_{2} e_{3}+b_{1} e_{1} e_{3} e_{5}-b_{1} e_{3}^{2} e_{4}-b_{2} e_{1}^{2} e_{3}-b_{2} e_{1} e_{4} e_{5}+b_{2} e_{3} e_{4}^{2}- \\
& -b_{3} e_{2}^{2} e_{5}+b_{3} e_{2} e_{4}^{2}+b_{3} e_{2} e_{5}^{2}+b_{3} e_{3}^{2} e_{5}-b_{3} e_{4}^{2} e_{5}+b_{4} e_{1}^{3}+b_{4} e_{1} e_{2} e_{5}-2 b_{4} e_{1} e_{3}{ }^{2}+b_{4} e_{1} e_{4}{ }^{2}- \\
& -b_{4} e_{1} e_{5}^{2}-2 b_{4} e_{2} e_{3} e_{4}+b_{4} e_{3} e_{4} e_{5}-2 b_{5} e_{1}^{2} e_{4}+b_{5} e_{1} e_{2} e_{3}-2 b_{5} e_{1} e_{3} e_{5}-b_{5} e_{2} e_{4} e_{5}+ \\
& +b_{5} e_{3}^{2} e_{4}+b_{5} e_{4}^{3}+2 b_{6} e_{1}^{2} e_{3}-b_{6} e_{1} e_{2} e_{4}+2 b_{6} e_{1} e_{4} e_{5}+b_{6} e_{2}{ }^{2} e_{3}-b_{6} e_{2} e_{3} e_{5}-b_{6} e_{3}{ }^{3}+ \\
& +b_{7} e_{1} e_{3}{ }^{2}-b_{7} e_{1} e_{4}^{2}+b_{7} e_{2} e_{3} e_{4}-b_{6} e_{3} e_{4}^{2}
\end{aligned}
$$

$$
\begin{aligned}
\boldsymbol{c h}_{4}= & 2 c_{1}^{2} e_{2}^{2}-5 c_{1}^{2} e_{2} e_{5}+9 c_{1}^{2} e_{4}^{2}+2 c_{1}^{2} e_{5}^{2}-6 e_{1} c_{1} c_{2} e_{2}+12 e_{1} c_{1} c_{2} e_{5}-18 c_{1} c_{2} e_{3} e_{4}- \\
& -18 c_{1} c_{3} e_{1} e_{4}+12 e_{3} c_{3} c_{1} e_{2}-6 e_{3} c_{3} c_{1} e_{5}-c_{2}{ }^{2} e_{2}{ }^{2}+c_{2}{ }^{2} e_{2} e_{5}+9 c_{2}{ }^{2} e_{3}{ }^{2}+2 c_{2}{ }^{2} e_{5}{ }^{2}- \\
& -18 c_{2} c_{3} e_{1} e_{3}-6 e_{4} c_{3} c_{2} e_{2}-6 e_{4} c_{3} c_{2} e_{5}+9 c_{3}{ }^{2} e_{1}^{2}+2 c_{3}{ }^{2} e_{2}{ }^{2}+c_{3}{ }^{2} e_{2} e_{5}-c_{3}{ }^{2} e_{5}{ }^{2}
\end{aligned}
$$

$$
\begin{gathered}
c h_{5}=b_{1}{ }^{4}-6 b_{1}{ }^{3} b_{5}+7 b_{2}{ }^{2} b_{1}{ }^{2}-36 b_{6} b_{2} b_{1}{ }^{2}-50 b_{3}{ }^{2} b_{1}{ }^{2}+57 b_{4}{ }^{2} b_{1}{ }^{2}-36 b_{4} b_{7} b_{1}{ }^{2}+b_{5}{ }^{2} b_{1}{ }^{2}+ \\
+57 b_{6}{ }^{2} b_{1}{ }^{2}+7 b_{7}{ }^{2} b_{1}{ }^{2}-36 b_{2}{ }^{2} b_{5} b_{1}+60 b_{3} b_{2} b_{4} b_{1}-40 b_{3} b_{2} b_{7} b_{1}+128 b_{6} b_{2} b_{5} b_{1}+150 b_{3}{ }^{2} b_{5} b_{1}+ \\
+160 b_{3} b_{6} b_{4} b_{1}+60 b_{3} b_{6} b_{7} b_{1}-206 b_{4}{ }^{2} b_{5} b_{1}+88 b_{4} b_{7} b_{5} b_{1}+24 b_{5}{ }^{3} b_{1}-136 b_{6}{ }^{2} b_{5} b_{1}-6 b_{7}{ }^{2} b_{5} b_{1}+ \\
+b_{2}{ }^{4}-6 b_{6} b_{2}{ }^{3}-50 b_{3}{ }^{2} b_{2}{ }^{2}+12 b_{2}{ }^{2} b_{4}{ }^{2}-6 b_{2}{ }^{2} b_{4} b_{7}+57 b_{2}{ }^{2} b_{5}{ }^{2}+b_{6}{ }^{2} b_{2}{ }^{2}+7 b_{2}{ }^{2} b_{7}{ }^{2}+ \\
+150 b_{3}{ }^{2} b_{2} b_{6}-340 b_{3} b_{2} b_{4} b_{5}+60 b_{3} b_{2} b_{7} b_{5}+4 b_{2} b_{4}{ }^{2} b_{6}-52 b_{2} b_{4} b_{7} b_{6}-136 b_{6} b_{2} b_{5}{ }^{2}+ \\
+24 b_{2} b_{6}{ }^{3}-6 b_{2} b_{7}{ }^{2} b_{6}+625 b_{3}{ }^{4}+200 b_{3}{ }^{2} b_{4}{ }^{2}+150 b_{3}{ }^{2} b_{7} b_{4}+200 b_{3}{ }^{2} b_{5}{ }^{2}+200 b_{3}{ }^{2} b_{6}{ }^{2}- \\
-50 b_{3}{ }^{2} b_{7}{ }^{2}-240 b_{3} b_{6} b_{4} b_{5}+160 b_{3} b_{6} b_{7} b_{5}+16 b_{4}{ }^{4}+24 b_{4}{ }^{3} b_{7}+137 b_{4}{ }^{2} b_{5}{ }^{2}+17 b_{6}{ }^{2} b_{4}{ }^{2}+ \\
+b_{4}{ }^{2} b_{7}{ }^{2}-76 b_{4} b_{7} b_{5}{ }^{2}+4 b_{6}{ }^{2} b_{4} b_{7}-6 b_{7}{ }^{3} b_{4}+16 b_{5}{ }^{4}+32 b_{6}{ }^{2} b_{5}{ }^{2}+12 b_{7}{ }^{2} b_{5}{ }^{2}+ \\
+16 b_{6}{ }^{4}+12 b_{6}{ }^{2} b_{7}{ }^{2}+b_{7}{ }^{4}
\end{gathered}
$$

Theorem 9. The set of the following thirteen invariants

$$
o d, d v_{1}, d v_{2}, d v_{3}, \operatorname{tr}_{1}, \operatorname{tr}_{2}, \operatorname{tr}_{3}, \operatorname{tr}_{4}, c h_{1}, c h_{2}, c h_{3}, c h_{4}, c h_{5}
$$

is a minimal generating set of the algebra $\mathbb{C}\left(U_{3}\right)^{\mathfrak{s l}_{2}}$.

Proof. It is enough to prove that the elements are algebraically independed. Let us consider the Jacobian $13 \times 16$-matrix of the polynomial set: 


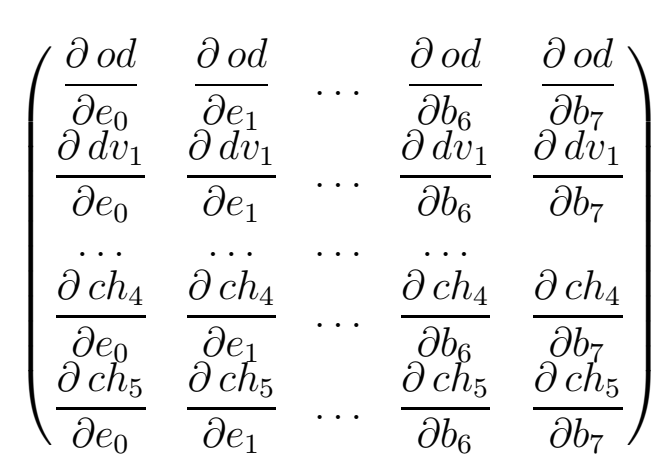

It is sufficient to show that the rank of the matrix is equal to 13. After substituting the following expressions

$$
\begin{aligned}
& e_{0}=1, e_{1}=1, e_{2}=23, e_{3}=53, e_{4}=97, e_{5}=151, b_{1}=541, b_{2}=661, b_{3}=827, \\
& b_{4}=1009, b_{5}=1193, b_{6}=1427, b_{7}=1619, c_{1}=227, c_{2}=311, c_{3}=419,
\end{aligned}
$$

into the Jacobian matrix, we get a matrix whose entries are all numbers. Then, by direct calculation, we obtain that its rankis equal to thirteen. It implies that the Jacobian matrix has the maximal rank equal to thirteen which proves the theorem.

Applying the same scheme, we can find the minimal generating sets of higher orders, for instance, the minimal generating sets of order four consists of 28 algebraically independent invariants.

\section{Conclusion}

In this article, we reviewed the 3D geometric moment invariants in the terms of the classical invariant theory. We divided all invariants into two types by introducing the notions of the

algebras of simultaneous rational and polynomial rotation invariants $\mathbb{C}[\eta]_{d}^{S O(3)}$ and $\mathbb{C}(\eta)_{d}^{S O(3)}$ up to order $d$ where $\eta$ is a set of normalized moments which are already invariants under the scaling and translations. In addition, we proved that these algebras are isomorphic to some classical object of the invariant theory, that is, to the algebras of join invariants of binary forms $\mathbb{C}\left[U_{d}\right]^{S L(2)}$ and $\mathbb{C}\left(U_{d}\right)^{S L(2)}$. Further on, we used Lie infinitesimal method and reduced the problem of calculating the invariants of the group $S O(3)$ to the equivalent one of calculating the invariants of the Lie algebra $\mathfrak{s l}_{2}$. From the computational point of view, it is much more simpler problem dealing with polynomial derivations.

In the rational case we count out the cardinality of the minimal generating set of the algebra $\mathbb{C}\left(U_{d}\right)^{S L(2)}$ and present such minimal generating set for invariants of the degrees two and three. Also we found the explicit form of the series of the invariants of the degree one of an arbitrary order, which plays an important role in different applications as a low-order moments which are less sensitive to noise than the higher-order ones.

The author hopes that the results will be useful to the researchers in the fields of image analysis and pattern recognition. Though, the geometric moments are not as effective as the orthogonal ones are, the obtained results are of independent theoretical interest.

As we have seen, in contrast to the $2 \mathrm{D}$ case, there is no satisfactory description of 3D rotational invariants of arbitrary order, and the problem of finding the basis of such invariants is hopeless. In our forthcoming researches, we are going to present another invariant constructions, which seems to be an effective way of describing of 3D image moments. 


\section{REFERENCES}

[1] Pratama, S.F., Muda, A.K., Choo, Y. et al. ATS drugs molecular structure representation using refined 3D geometric moment invariants. J Math Chem 55, 1951-1963 (2017).

[2] Abdalbari, A., Ren, J. Green, M. Seeds classification for image segmentation based on 3-D affine moment invariants. Biomed. Eng. Lett. 6, 224-233 (2016).

[3] Zucchelli M/ , Deslauriers-Gauthier S., Deriche R., A computational Framework for generating rotation invariant features and its application in diffusion MRI, Medical Image Analysis(60)101597,(2020)

[4] Kushwaha, A.K.S., Srivastava, S., Srivastava, R. Multi-view human activity recognition based on silhouette and uniform rotation invariant local binary patterns. Multimedia Systems 23, 451-467 (2017).

[5] Sadjadi, F. A., Hall, E. L. (1980). Three-Dimensional Moment Invariants. IEEE Transactions on Pattern Analysis and Machine Intelligence, PAMI-2(2), 127-136.

[6] Lo C.-H., Don H.-S., 3-D moment forms: their construction and application to object identification and positioning, IEEE Trans. Pattern Anal. Mach. Intell. 11 (1989) 1053-1064.

[7] Suk T., Flusser J., Boldyš J., 3D rotation invariants by complex moments, Pattern Recognition, Volume 48, Issue 11,2015, Pages 3516-3526.

[8] Flusser J., Suk T., Zitová B., 2D and 3D Image Analysis by Moments. John Wiley and Sons (2017)

[9] Suk T., Flusser J. (2011) Tensor Method for Constructing 3D Moment Invariants. In: Real P., Diaz-Pernil D., Molina-Abril H., Berciano A., Kropatsch W. (eds) Computer Analysis of Images and Patterns. CAIP 2011. Lecture Notes in Computer Science, vol 6855. Springer, Berlin, Heidelberg

[10] Burel G., H. Hhocq H., " Three-dimensional invariants and their application to object recognition" Signal Processing, 45 (1995) l-22.

[11] Hu M. K.: Visual pattern recognition by moment invariants. IRE Trans. Inform. Theory. 8(2), 179-187 (1962)

[12] Bedratyuk, L. 2D Geometric Moment Invariants from the Point of View of the Classical Invariant Theory. J Math Imaging Vis (2020). doi:10.1007/s10851-020-00954-9

[13] Marc Olive, About Gordan's algorithm for binary forms, arXiv: 1403.2283v5 [math.RT], 2015

[14] Woit P., Quantum Theory, Groups and Representations, Springer, 2014, pp. 668,

[15] Hall B., Quantum Theory for Mathematicians, Springer, 2013, pp. 566

[16] Bedratyuk L., The Maple package for $S L_{2}$-invariants and kernel of Weitzenböck derivations, arXiv:1101.0622v1, 2011

[17] Bedratyuk L., The Maple package for calculating Poincaré series, arXiv:1006.5372, 2010.

[18] Fulton, W., Harris, J.: Reptesentation theory: a first course. Springer-Verlag New York (1991)

Khmelnitskiy nAtional University, Instytus'Ka, 11, KhmELnitskiy, 29016, UkRAine 